\title{
Lateral nasal advancement flap for reconstruction of the nasal sidewall and dorsum
}

\author{
Yutaka Ogawa ${ }^{1}$, Yasuko Ogawa ${ }^{2}$ \\ ${ }^{I}$ Department of Plastic and Reconstructive Surgery, Kansai Medical University, Osaka; ${ }^{2}$ Division of Dermatology, Ogawa Skin Clinic, Kyoto, \\ Japan
}

Malignant skin tumors and precancerous lesions have a predilection to be located in the nasal dorsum or sidewall. Although invasive reconstructions have been presented, no simple and suitable method has yet been reported for this area. The flap presented herein, named the lateral nasal advancement flap, is designed on the adjacent lateral region of the sidewall or nasal dorsum and advanced in the medial direction. Two Burow's triangles are removed in the upper and lower portions of the flap: the upper triangle along the nasofacial sulcus and the lower triangle along the nasofacial sulcus and/or the alar groove. Excellent results were obtained in the two clinical cases described in this report. Neither a trap door deformity nor dog-ears developed in either case. The postsurgical scars followed the aesthetic lines and became inconspicuous. A distinct angle was formed in the nasofacial sulcus without anchor sutures. This surgical procedure is technically simple and is performed under local anesthesia. Although the flap is a cheek-based advancement flap, postsurgical scars do not remain in the cheek; instead, they are located in the nasofacial sulcus and alar groove. The lateral nasal advancement flap is recommended for reconstruction of the nasal sidewall and dorsum.

Keywords Lateral nasal advancement flap / Nasal sidewall reconstruction / Nasal dorsum reconstruction

\author{
Correspondence: Yutaka Ogawa \\ Department of Plastic and \\ Reconstructive Surgery, Kansai \\ Medical University, 6-2-1809 \\ Chagasaki, Osaka 520-0023, Japan \\ Tel: +81-77-572-5772 \\ Fax: +81-77-572-5772 \\ E-mail: ytk-ogawa@nifty.com
}

Received: September 5, $2019 \bullet$ Revised: October 10, $2019 \bullet$ Accepted: October 22, 2019

pISSN: 2234-6163 • elSSN: 2234-6171 • https://doi.org/10.5999/aps.2019.01193• Arch Plast Surg 2020;47:102-105

\section{INTRODUCTION}

Malignant skin tumors and precancerous lesions have a predilection to be located in the nasal dorsum or sidewall. A new application of a local advancement flap is proposed in this report. This flap, which the authors have named the lateral nasal advancement flap, is designed on the sidewall to the cheek to repair the sidewall or nasal dorsum by horizontal advancement. The flap is useful for the reconstruction of small to mid-sized skin defects of the dorsal nasal and sidewall region in a singlestage operation with excellent results. The procedure is per- formed under local anesthesia. As the flap draws upon conventional advancement flap techniques, the positioning of the $\mathrm{Bu}$ row's triangles on the nasofacial sulcus and alar groove is the most important technical point.

\section{IDEA}

Two horizontal incisional lines are marked from the upper and lower edge of the postsurgical defect on the nasal dorsum or sidewall, and a rectangular flap composed of two incisions and the lateral edge of the defect is then designed. Two triangle- 
shaped sections of skin are removed in the upper and lower portions of the flap: the upper triangle along the nasofacial sulcus and the lower triangle along the nasofacial sulcus and alar groove, thereby eliminating the dog-ears that would otherwise result (Fig. 1). The length of the triangle-base depends on the horizontal length and position of the defect and the laxity of the skin. A rectangular flap composed of the remaining sidewall and cheek skin is designed. The flap is advanced horizontally to the defect with the upper triangular excision running right along the nasofacial sulcus, with the lower excision coinciding with the nasofacial sulcus and/or alar groove (Fig. 2). The maximum size of the defect to be repaired is limited to a horizontal length of approximately $2 \mathrm{~cm}$ and a vertical length of roughly $2.5 \mathrm{~cm}$. Although the flap does not include a main artery, as in an axial pattern flap, its vascularity is guaranteed by the vascular network. The resulting postsurgical scars become inconspicuous because the major portions of the excisional lines coincide with aesthetic borders. If a defect is large and incorporates both sidewalls of the nose, a double opposing lateral nasal advancement flap is possible.

\section{Case 1}

A 91-year-old woman presented with an erythematous lesion on her right nasal sidewall that had been present for 1 year preoperatively. A pathological examination revealed solar keratosis. The lesion was removed with a 5-mm safety margin and a lateral nasal advancement flap was designed for the reconstruction of the $25 \times 18 \mathrm{~mm}$ (height $\times$ width) skin defect on the right nasal sidewall. The postoperative scars were minimal and not conspicuous. No recurrence was observed 2 years after the operation. The results were excellent (Fig. 3).

\section{Fig. 1. Design of the lateral nasal advancement flap}

Two Burow's triangles (yellow) are removed along the nasofacial sulcus and/or alar groove.

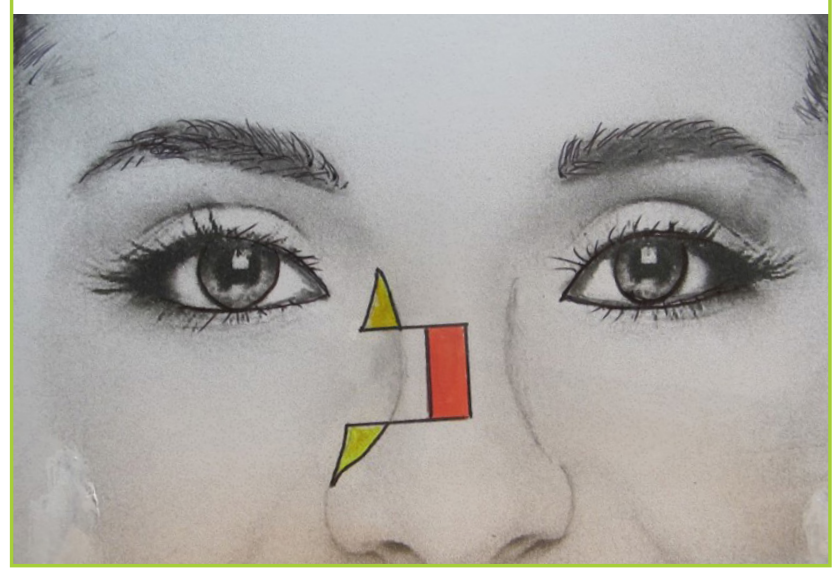

\section{Case 2}

A 78-year-old woman suffered from solar keratosis in her right sidewall. The final defect measured $21 \times 18 \mathrm{~mm}$ after removal, and a lateral nasal advancement flap was raised for the reconstruction. The postoperative results were excellent (Fig. 4).

\section{DISCUSSION}

Although many methods currently exist for reconstruction of the sidewall or nasal dorsum, these procedures can often lead to conspicuous scarring or deformities. An axial frontonasal flap inevitably results in a major scar [1]. A nasolabial subcutaneous pedicle flap is an island flap; therefore, a trap door deformity may develop, potentially requiring a secondary operation. The Rintala flap is recommended for horizontally wide defects, but not for narrow defects. The disadvantages of the Rintala flap include long scars, flatness of the frontonasal angle, and the risk of a nasal tip deformity or short nose [2].

In terms of distortion after flap transposition, it is preferable to transfer a flap from the horizontal direction to the vertical direction [3]. The advancement perforator cheek flap by Rossi et al. [4] requires a long incision along the nasofacial sulcus and melolabial crease in the lower portion and another long incision along the lower lid margin in the upper portion, so that the lower lid margin may result in distortion of the lower lid. The lateral nasal advancement flap forms neither dog-ears nor a trap door deformity, as the triangular sections of excess skin are removed along the nasofacial sulcus and alar groove. The resulting scars are inconspicuous because they coincide with the aesthetic lines.

The lateral nasal advancement flap has the following advantages: (1) A distinct angle is formed in the nasofacial sulcus without the anchor sutures; (2) Postsurgical scars occur along the aesthetic lines or straight lines, and become inconspicuous; (3)

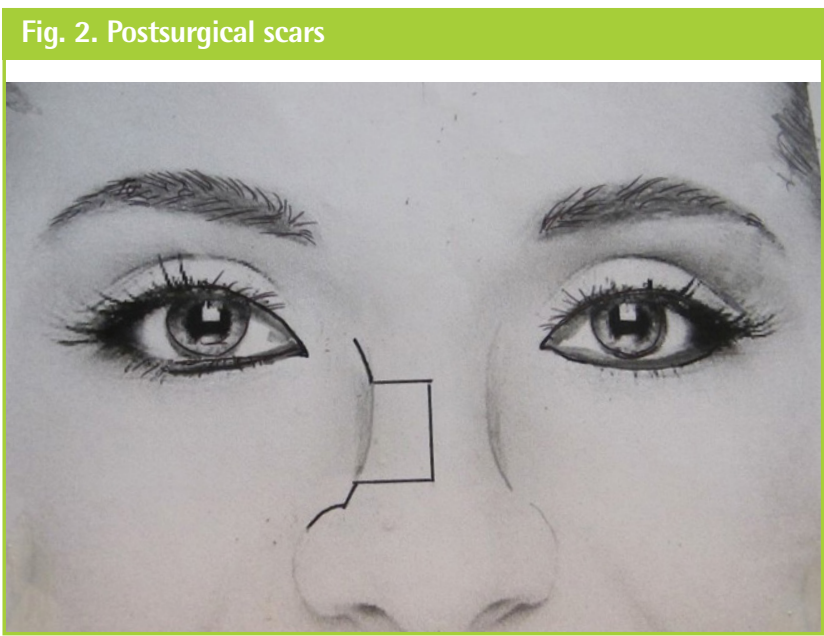




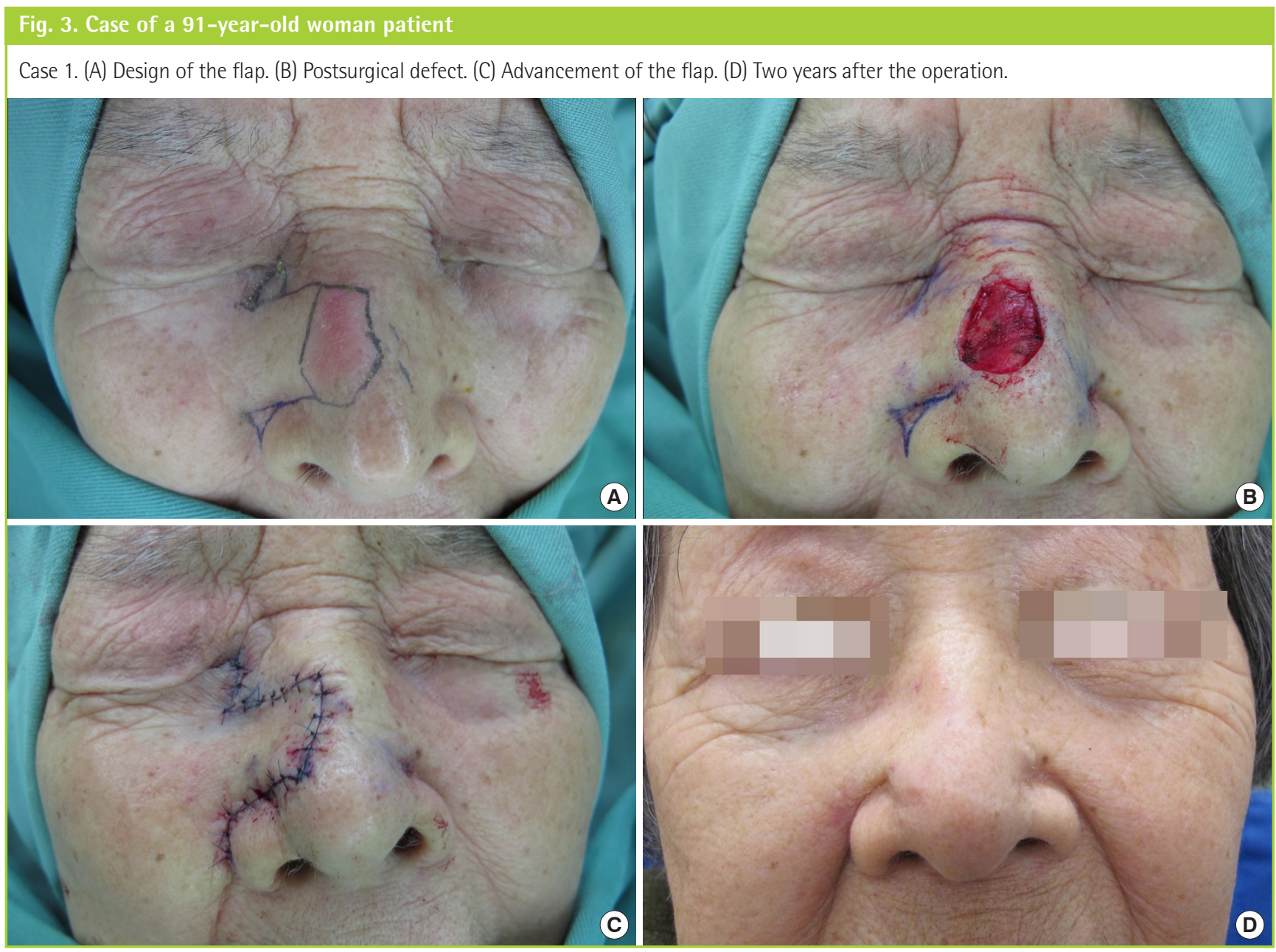

Fig. 4. Case of a 78-year-old woman patient

Case 2. (A) Design of the flap. (B) Two years after the operation.

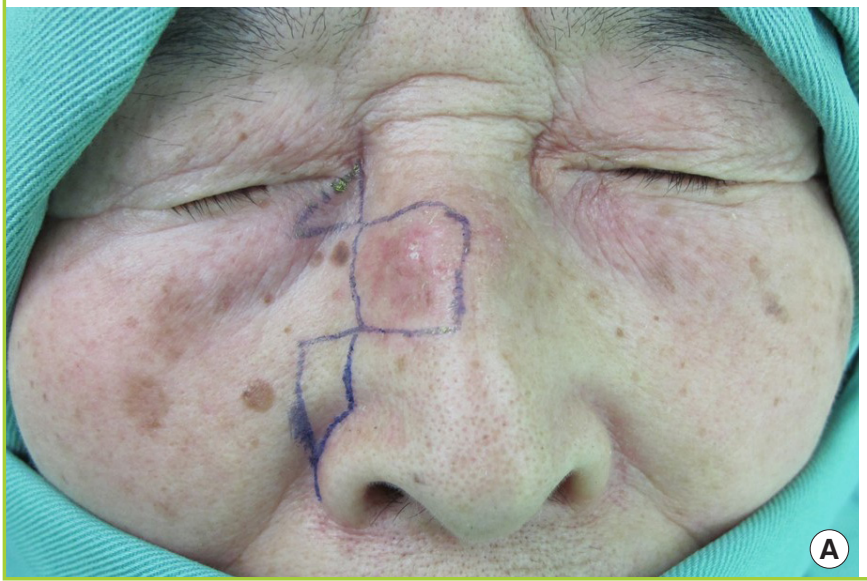

The surgical procedure is technically simple; (4) Although the flap is a cheek-based advancement flap, postsurgical scars do not remain in the cheek, but are located in the nasofacial sulcus and alar groove; or (5) A double opposing lateral nasal advancement flap is an option for a large defect.

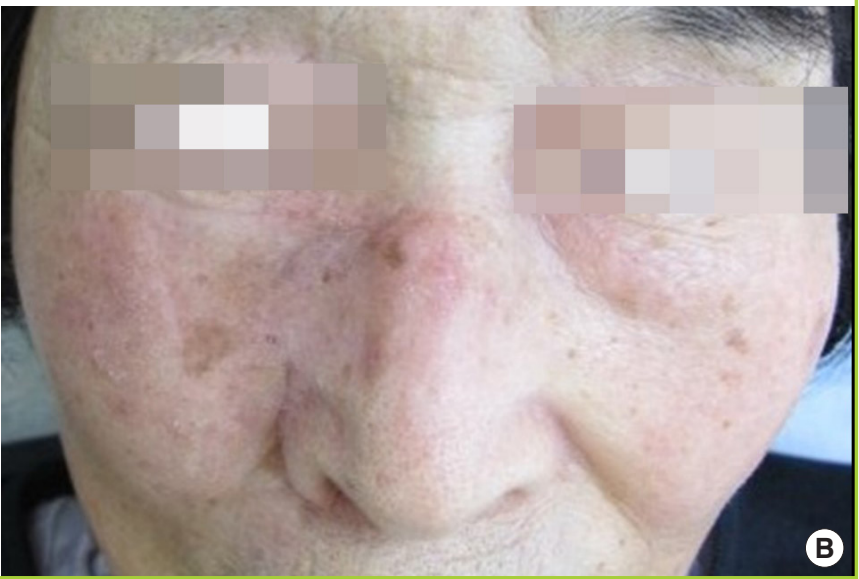

The disadvantages are as follows: (1) The area to be reconstructed is limited by its proximity to the inner canthus. The upper area of the defect needs to be at least several millimeters below the level of the inner canthus. The lower limit of the defect to be reconstructed is the top of the alar groove; (2) The maxi- 
mum size to be reconstructed is generally limited to about 20 $\mathrm{mm}$ in width $\times 25 \mathrm{~mm}$ in height for a single flap.

If an adequate length of the Burow's base is obtained and cases are selected properly, this flap will be available for young patients. This procedure is a valid option for older patients with sufficient skin redundancy.

\section{NOTES}

\section{Conflict of interest}

No potential conflict of interest relevant to this article was reported.

\section{Ethical approval}

The study was approved by the Institutional Review Board of Kansai Medical University (IRB No. 2016553) and performed in accordance with the principles of the Declaration of Helsinki. Written informed consents were obtained.

\section{Patient consent}

The patients provided written informed consent for the publication and the use of their images.

\section{Author contribution}

Conseptualization: Ogawa Yu, Ogawa Ya. Data curation: Ogawa
Yu. Formal analysis: Ogawa Yu. Methology: Ogawa Yu. Project administration: Ogawa Yu, Ogawa Ya. Visualization: Ogawa Yu. Writing-original draft: Ogawa Yu. Writing-review \& editing: Ogawa Yu.

\section{ORCID}

Yutaka Ogawa https://orcid.org/0000-0002-3384-6876

Yasuko Ogawa https://orcid.org/0000-0002-4890-9606

\section{REFERENCES}

1. Marchac D, Toth B. The axial frontonasal flap revisited. Plast Reconstr Surg 1985;76:686-94.

2. Rintala AE, Asko-Seljavaara S. Reconstruction of midline skin defects of the nose. Scand J Plast Reconstr Surg 1969;3: 105-8.

3. Ogawa Y, Takemoto T, Suzuki K, et al. Application of the lateral orbital flap to reconstruction of the upper and lower eyelids and the eye socket after enucleation. Ann Plast Surg 2011;66:360-3.

4. Rossi M, Milia A, Carmisciano M, et al. Advancement perforator cheek flap for aesthetic one-stage reconstruction of postoncological extended split-thickness defects of the nasal sidewall. ScientificWorldJournal 2013;2013:169208. 